\title{
Study design III: Cross-sectional studies
}

\author{
Kate Ann Levin \\ Dental Health Services Research Unit, University of Dundee, Dundee, Scotland, UK \\ In this series, I previously gave an overview of the main types of study design and \\ the techniques used to minimise biased results. Here, I describe cross-sectional \\ studies, their uses, advantages and limitations. \\ Evidence-Based Dentistry (2006) 7, 24-25. doi:10.1038/sj.ebd.6400375
}

Cross-sectional studies are carried out at one time point or over a short period (Figure 1). They are usually conducted to estimate the prevalence of the outcome of interest for a given population, commonly for the purposes of public health planning. Data can also be collected on individual characteristics, including exposure to risk factors, alongside information about the outcome. In this way crosssectional studies provide a 'snapshot' of the outcome and the characteristics associated with it, at a specific point in time.

\section{Why carry out a cross-sectional study?}

A cross-sectional study design is used when

- The purpose of the study is descriptive, often in the form of a survey. Usually there is no hypothesis as such, but the aim is to describe a population or a subgroup within the population with respect to an outcome and a set of risk factors.

- The purpose of the study is to find the prevalence of the outcome of interest, for the population or subgroups within the population at a given timepoint.

Cross-sectional studies are sometimes carried out to investigate associations between risk factors and the outcome of interest. They are limited, however, by the fact that they are carried out at one time point and give no indication of the sequence of events - whether exposure occurred before, after or during the onset of the disease outcome. This being so, it is impossible to infer causality.

The next four publications of Evidencebased Dentistry describe other study designs that may be more appropriate for the purposes of understanding associations between exposure to risk factors and the outcome of interest. Nevertheless, cross-sectional studies indicate associations that may exist and are therefore useful in generating hypotheses for future research.

Repeated cross-sectional studies may be carried out to give a pseudolongitu-

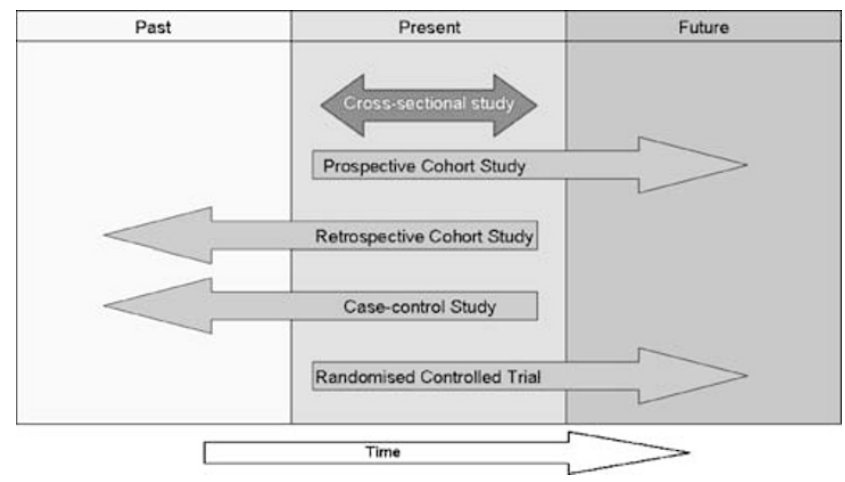

Figure 1. Cross-sectional studies.

dinal study, where the individuals included in the study are either chosen from the same sampling frame or from a different one. An example might be the British Association for the Study of Community Dentistry Survey in which 5-year-old children are examined annually and prevalence of caries is recorded. The prevalence of caries for this age group is monitored over time and this information is used in public health policy planning and in the development of targeting strategies.

\section{Sample selection and response rates}

The sample frame used to select a sample and the response rate determine how well results can be generalised to the population as a whole. The sample used in a large cross-sectional study is often taken from the whole population. This is the optimum situation: if the sample is selected using a random technique it is likely that it will be highly representative. In order for the results to be representative of the population, however, not only must the selected sample be representative but so must the responders. Nonresponse is a common problem in wide-scale surveys; techniques to minimise nonresponse include telephone and mail prompting, second and third mailing of surveys, letters outlining the importance of replying and a range of incentives.

The level of nonresponse is one concern, but a greater one still is that of biased response, where a person is more likely to respond when they have a particular characteristic or set of characteristics. Bias will occur when the characteristic in question is in some way related to the probability of having the outcome. The response rate of a survey conducted by door-to-door interview looking at a particular disease, for example, may be highest in the elderly and unemployed because these groups are 
more likely to be in their home during the day. These two groups are also more likely to experience higher levels of disease, therefore biasing the results.

\section{Measures of outcome and exposure}

A lot of information can be collected about potential risk factors in a crosssectional study. Loss to follow-up is a common concern in longitudinal studies and one of the strategies used to overcome this is to minimise the amount of information collected. This is not a problem in cross-sectional study design.

It is advisable to think carefully about what might be relevant because this is a good opportunity to gain a broad base of knowledge about subjects who have/do not have the outcome of interest, but it is also important to maintain optimum response levels. Associations between outcomes and exposures of long duration are particularly difficult to establish using cross-sectional studies.

\section{Advantages of cross-sectional studies}

- Relatively inexpensive and takes up little time to conduct;

- Can estimate prevalence of outcome of interest because sample is usually taken from the whole population;

- Many outcomes and risk factors can be assessed;

- Useful for public health planning, understanding disease aetiology and for the generation of hypotheses;

- There is no loss to follow-up.
Disadvantages of cross-sectional studies

- Difficult to make causal inference;

- Only a snapshot: the situation may provide differing results if another timeframe had been chosen;

- Prevalence-incidence bias (also called Neyman bias). Especially in the case of longer-lasting diseases, any risk factor that results in death will be under-represented among those with the disease.

\section{Recommended reading}

1. Bland M. An Introduction to Medical Statistics. 3rd Edn. Oxford: Oxford University Press; 2001.

2. Pine CM, Pitts NB, Nugent ZI. British Association for the Study of Community Dentistry (BASCD) guidance on sampling for surveys of child dental health. A BASCD coordinated dental epidemiology programme quality standard. Commun Dent Health 1997; 14(Suppl 1):S10-S17. 